\title{
Offres De Soins Et Qualité De Vie Des Professionnels (Les) Du Sexe Sous ARV Les Communes De Marcory Et San-Pedro En Côte d'Ivoire
}

\author{
Dr. Koné Moussa (PhD, MA) \\ Socio-anthropologue, Enseignant-chercheur à l'Université Jean \\ LOROUGNON GUEDE de Daloa, Daloa, Côte d'Ivoire
}

Dr. Tanoh Say Clémentine (PhD, MA)

Sociologue de la Santé/ Socio-Gérontologue, Enseignant-chercheur à l'Université Jean LOROUGNON GUEDE de Daloa, Daloa, Côte d'Ivoire

Doi:10.19044/esj.2019.v15n5p265 ～URL:http://dx.doi.org/10.19044/esj.2019.v15n5p265

Résumé

Cette étude a porté sur les PVVIH au niveau des professionnelles du sexe en Côte d'Ivoire, singulièrement dans les communes de Marcory et San Pedro. Malgré les politiques mises en œuvre au niveau international et national en matière de lutte contre le VIH/SIDA, le taux de contamination ne fait que s'accroître. Ainsi, comment les offres de soins de santé à l'endroit des personnes vivant avec le VIH influencent-elles la qualité de vie des professionnels (les) du sexe sous ARV ? Pour répondre à cette interrogation, l'approche qualitative a été mobilisée. Elle a été réalisée avec un échantillon de 25 professionnels du sexe recrutés à l'aide de l'échantillonnage par réseaux ou en boule de neige. Avec ce groupe cible composé de 8 hommes et de 17 femmes dont l'âge varie de 10-13 à 27 ans et plus, des entrevues approfondies ont été menées. Aussi, 05 représentants de la population experte ont été interviewés. Il en ressort que les représentations sociales des ARV expliquent la non observance du traitement. De la même manière, les itinéraires thérapeutiques, l'environnement social et les difficultés économiques influencent l'état de santé physique et psychologique des PS vivant avec le VIH. Il est aussi pointé du doigt les effets secondaires des médicaments. Leur stigmatisation les encourage à une sorte d'auto-victimation qui les éloigne de plus en plus des traitements antirétroviraux. Malgré tout, une radiographie du système de santé en la matière y compris les dispositifs médicaux est à revoir pour mieux aborder la prise en charge de ces malades.

Mots-clés : PVVIH, Professionnelles du sexe, Offres de soins, Qualité de vie 


\title{
Offers of Care and Quality of Life of the Sexworkers Under ARV Communes of Marcory and San-Pedro in Côted'Ivoire
}

\author{
Dr. Koné Moussa (PhD, MA) \\ Socio-anthropologue, Enseignant-chercheur à l'Université Jean \\ LOROUGNON GUEDE de Daloa, Daloa, Côte d'Ivoire \\ Dr. Tanoh Say Clémentine (PhD, MA) \\ Sociologue de la Santé/ Socio-Gérontologue, Enseignant-chercheur à \\ l'Université Jean LOROUGNON GUEDE de Daloa, Daloa, Côte d'Ivoire
}

\begin{abstract}
This paper focuses on VPHIV at the level of sex workers in Côte d'Ivoire, particularly in the communes of Marcory and San Pedro. Despite the policies implemented at the international and national level in the fight against HIV/AIDS, the rate of transmission is increasing. So, how does the health care offered to people living with HIV influence the quality of the life of ARV sex workers? To answer this question, the qualitative approach was employed in this study. It was conducted with a sample of 25 sex workers recruited using network or snowball sampling. With this target group of 8 men and 17 women ranging in age from 10-13 to 27 years and over, in-depth interviews were conducted. Also, 05 representatives of the expert population were interviewed. It appears that the social representations of ARVs explain the non-observance of the treatment. Also, therapeutic routes, the social environment, and economic difficulties have a significant positive influence on the physical and psychological health of people living with HIV. Consequently, the side effects of the drug were pointed out. The stigmatization makes them self-victimized, which moves them away from antiretroviral therapy. Nevertheless, a radiography of the health system in this area, including medical devices, should be reviewed to better address the care of these patients.
\end{abstract}

Keywords: VPHIV, Professional Sex Workers, Caregiving, Quality of Life

\section{SIGLES}

AGR

Activités Génératrices de Revenu

APROSAM

Association pour la Promotion de la Santé de la Femme, de la Mère, de l'Enfant et de la Famille

ARV

EGF

(des médicaments) Anti Rétroviraux

Entretien de Groupe Focalisé 


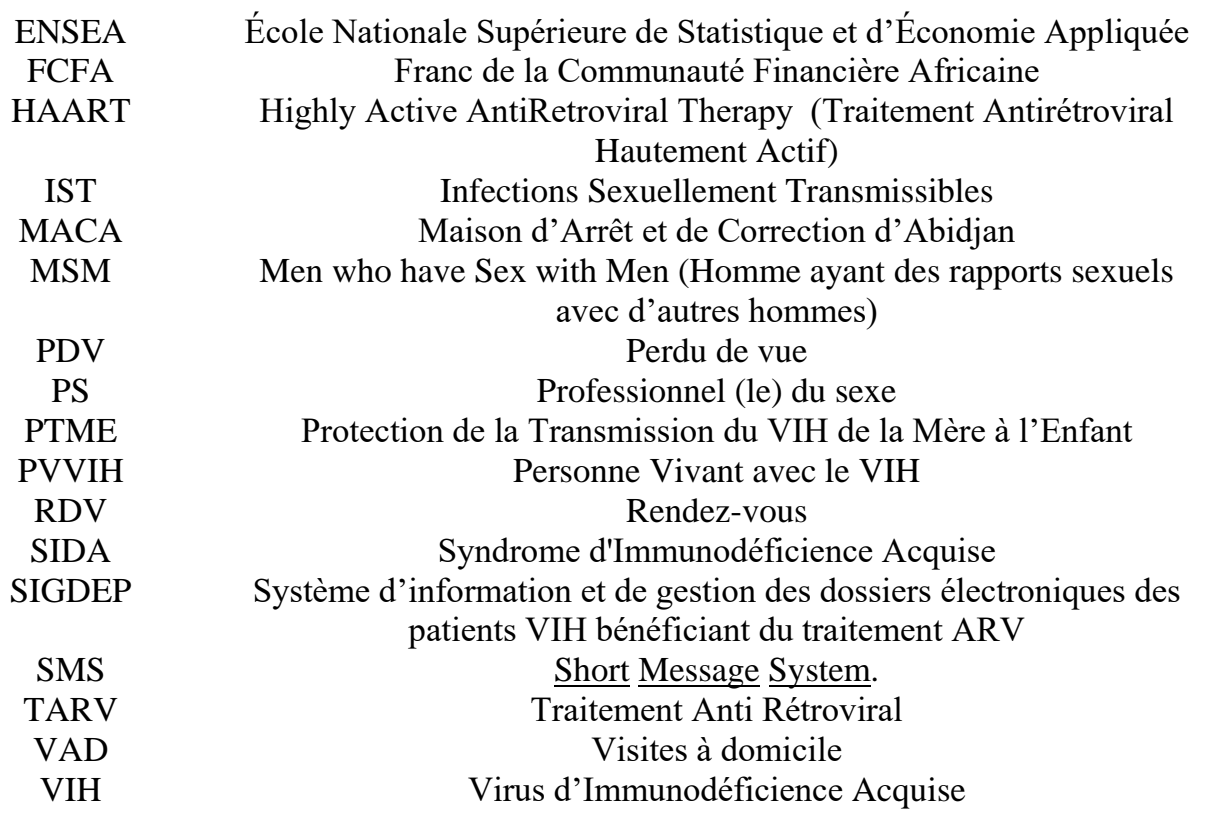

\section{Introduction}

Malgré le dispositif institutionnel de sensibilisation et de prise en charge gratuite des PVVIH+, notamment des PS PVVIH+ (Carillon, 2010), un tiers des professionnels du sexe sortent du circuit des soins alors que la faible rétention des PS sous ARV aura un impact sur la population générale. En effet, les travaux conduits dans plusieurs pays en Afrique Sub-saharienne y compris en Côte d'Ivoire indiquent une prévalence de l'infection à VIH 10-20 fois plus élevée chez les personnes avec de multiples partenaires sexuels par rapport à la population des femmes enceintes. Selon les mêmes sources, le risque de transmission d'une PS infectée peut augmenter de l'ordre de 100 fois en cas du nombre élevé de clients, facteur de charge virale importante de VIH. En outre, les PS infectées par le VIH et souffrant d'autres IST (plus particulièrement les IST ulcératives dont, entre autres, le HSV-2, le chancroïde, ...) sont plus susceptibles de transmettre le VIH notamment dans les milieux à faible taux de circoncision masculine. Ces maladies opportunistes qui nécessitent une prise en charge médicale spécifique sont susceptibles de miner la qualité de vie des professionnels (les) du sexe.

La qualité de vie est définie comme "la perception qu'a un individu de sa place dans l'existence, dans le contexte de la culture et du système de valeurs dans lesquelles il vit, en relation avec ses objectifs, ses attentes, ses normes et ses inquiétudes » (OMS, 1993). Telle que définie, la qualité de vie endosse un caractère holistique parce qu'elle inclut la représentation que l'individu se fait de sa place en société, inférant une relation implicite entre santé physique et psychologique, l'affectivité, les besoins, les valeurs, le 
réseau social et l'autonomie en rapport avec des facteurs environnementaux spécifiques (Cuchet, 2011). Ce concept renvoie donc à de multiples dimensions, tant objectives que subjectives : l'état physique (l'autonomie et les capacités physiques; les sensations somatiques : symptômes, conséquence des traumatismes ou des procédures thérapeutiques, douleurs), l'état psychologique (émotivité, anxiété, dépression) et le statut social (relations sociales et rapport à l'environnement familial, amical ou professionnel). Ces dimensions de la qualité de vie sont retenues dans cette étude, ce qui nous amène à nous poser la question suivante : Comment les offres de soins de santé à l'endroit des personnes vivant avec le VIH influencent-elles la qualité de vie des professionnels (les) du sexe sous ARV? De façon spécifique, les itinéraires thérapeutiques choisis par les professionnels (les) du sexe ne justifient-ils pas leur qualité de vie ? Cette étude vise alors à analyser la qualité de vie des professionnels du sexe sous ARV, en rapport aux offres de soins de santé.

Également, elle est structurée autour de l'hypothèse suivante: la qualité de vie des professionnels du sexe sous ARV est fonction des offres de soins de santé.

\section{I : La méthodologie de recherche \\ - Site et population}

La présente étude s'est déroulée dans les communes de Marcory et San-Pedro. Cette étude a eu lieu en 2017 et elle a duré trois (3) mois.

Marcory est une commune de la ville Abidjan, en Côte d'Ivoire. Elle est située au sud du District d'Abidjan entre la commune de Treichville et celle de Koumassi, sur 1'île de Petit Bassam. Elle est composée de 12 quartiers et de 3 villages. Marcory est une grande plateforme commerciale avec des supers et hypermarchés, ses entreprises et ses restaurants. Sa population est estimée à 500000 habitants (RGPH, 2014).

La ville de San-Pedro est située à $9^{\circ} 32$ de latitude nord et $6^{\circ} 29$ de longitude ouest $\left(\underline{4^{\circ} 51^{\prime} \mathrm{N}, 6^{\circ} 42^{\prime} \mathrm{O}}\right)$, et elle fait partie de la région du BasSassandra. Elle est située au bord de l'océan Atlantique, sur le golfe de Guinée, à $348 \mathrm{~km}$ d'Abidjan, capitale économique du pays. La population de San Pedro est estimée à 420000 habitants.

Trois raisons fondamentales justifient le choix de ces communes. D'abord, de par leurs sites géographiques, Marcory et San-Pedro présentent des caractéristiques singulières. Marcory est à cinq (5) minutes de la commune du Plateau, centre des affaires ; à 5 minutes de Cocody par le troisième pont et à 5 minutes de l'aéroport et également par ses atouts (pôle industriel, échangeur, grands restaurants, centres commerciaux...), cette commune attire les opérateurs économiques, de même que les professionnels du sexe. De même, la ville de San-Pedro est bercée par le Golfe de Guinée qui lui offre ses 
150 kilomètres de plages, et elle dispose aussi d'infrastructures balnéaires et de réceptifs hôteliers de qualité.

Ensuite, certains quartiers de Marcory comme Zone 4 et Biétry sont habités par des hommes d'affaires et des expatriés. Dans ces lieux, des constats empiriques laissent entrevoir une forte concentration de professionnels du sexe.

Le district du Bas-Sassandra est l'un des plus riches du pays, notamment grâce au port de San-Pedro qui est le second de la Côte d'Ivoire après celui d'Abidjan (et premier terminal cacaoyer au monde). Il est le premier port mondial pour les exportations de fèves de cacao avec plus de la moitié de la récolte ivoirienne (1,8 million de tonnes) qui y transite.

Enfin, selon une étude effectuée sur « la prostitution pratiquée à ciel ouvert à Abidjan », Marcory fait partie des communes réputées dans ce domaine. Et, selon le CriAbidjan.net de septembre 2014, la prostitution prend une ampleur de plus en plus inquiétante à San Pedro, 2ème port de la Côte d'ivoire.

L'approche qualitative a été mobilisée pour la présente étude. Elle a été réalisée avec un échantillon de 25 professionnels du sexe recrutés à l'aide de l'échantillonnage par réseaux ou en boule de neige. Nous avons eu contact avec l'un d'eux grâce à l'ONG Alliance de Côte d'Ivoire et à partir de ce dernier, nous avons recruté un second puis de nouveaux autres participants selon les recommandations faites par les premiers participants sélectionnés. Les personnes ainsi proposées ont été ensuite interviewées et il leur a été demandé de proposer d'autres personnes encore. Le processus est répété jusqu'à satiété, c'est-à-dire jusqu'à ce qu'aucun nouveau nom ne soit donné. Les rencontres ont eu lieu dans des endroits très discrets, à la suite de négociations de plusieurs rendez-vous reportés à maintes reprises.

\section{- La production des données de terrain}

\section{- Les entretiens approfondis}

Nous avons pu faire des entrevues approfondies avec le groupe cible composé de 8 hommes et de 17 femmes dont l'âge varie de 10-13 à 27 ans et plus. Ces professionnels du sexe viennent de toutes les communes du district d'Abidjan. Certains soutiennent qu'ils préfèrent « travailler » hors de leurs communes et quartiers d'habitation afin d'être à l'abri des regards malveillants de leurs connaissances. Les entretiens ont porté sur les thèmes suivants : la représentation sociale des soins de santé, les itinéraires thérapeutiques, l'état de santé physique et psychologique, les rapports à l'environnement social.

En plus de la population cible, la population experte a été interviewée. Cette dernière est composée de directeurs généraux et régionaux du Ministère de la Santé et de l'Hygiène Publique et de directeurs des structures sanitaires, 
des ONG et autres services intervenant dans le domaine du VIH, soit environ une vingtaine de personnes interrogées.

Les entretiens ont porté essentiellement sur les soins de santé offerts aux PVVIH+ en général et aux PS PVVIH+ en particulier.

\section{- Études de cas (case studies) comme stratégie de recherche}

La mobilisation de cette stratégie de recherche consiste à faire, dans le cadre de cette étude, une radioscopie des professionnels du sexe et de la prostitution. Elle a permis d'observer les acteurs en interaction et de repérer, par la technique de «snowball sampling», tous les acteurs ou instances impliqués dans le fait étudié. À l'image de l'enquête systémique par questionnaire, l'étude de cas permet l'observation fine, par exemple, du périple et des "frasques" du professionnel du sexe... Cela concerne également la place des professionnels du sexe au sein de leur famille, dans leur communauté, leur profil, leur niveau d'étude, leur statut, ce qui permet la compréhension de leurs actions.

Pour cette étude, nous avons opté pour l'étude de cas sélectionnés pour leur caractère illustratif. L'étude de cas illustratifs est la description d'un cas ou de quelques cas significatifs dont l'objectif est de comprendre de façon empirique le ou les phénomènes qu'on observe.

\section{- L'analyse des données de terrain}

L'analyse des données s'est faite par une approche stratégique et « jeu » des acteurs (Crozier \& Friedberg, 1977). En outre, nous avons eu recours à l'approche ethnographique de terrain qui s'intéresse particulièrement à la description et à l'interprétation des comportements des groupes d'acteurs ou du système social étudié (Creswell, 1998). L'ethnographie, description ou description épaisse (Geertz, 1973) est une approche empirique qui consiste à faire une étude fine, détaillée, in situ, transversale et contextualisée des phénomènes observés dont l'objectif est une compréhension plus approfondie des interactions et des systèmes d'actions concrets (Crozier \& Friedberg, 1977).

\section{II : Les résultats de l'étude}

II-1 : Les représentations sociales des soins de santé par les PS sous ARV II-.1.1 : Les sensations somatiques ou Perceptions du traitement ARV chez les PS

\section{- . Se sentir guéri}

L'appropriation de la maladie, c'est le fait que le malade accepte sa sérologie et cherche vaille que vaille à guérir systématiquement (Baldé, 2013). Dans ce sens, certains PS s'orientent vers les ARV mais au bout d'un certain temps, ils arrêtent le traitement lorsqu'ils constatent une amélioration de leur 
état de santé, c'est-à-dire, lorsqu'ils se sentent guéris. Une PS qui a arrêté son traitement témoigne : "Il y a eu un moment j'ai arrêté de prendre et je ne venais plus à la clinique. Près de 6 mois, et nous sommes dans le septième mois maintenant. Je me suis dit que j'étais guérie ». (Une PS de la Clinique de Confiance).

Il y a donc chez les PS cette envie d'arrêter le traitement parce qu'ils se sentent en bonne santé. Souvent, ils pensent même que cet état de bonne santé peut être maintenu continuellement sans ARV. Un exemple nous est rapporté par une PS PDV: «Comme elle a pris (les ARV), elle voit que ça va, elle croit que ça va continuer comme ça aussi or elle a laissé ». (Une PS PDV du centre Espérance).

En termes d'analyse, il y a une sorte de rejet de la maladie chronique qui fait que les patients sous ARV n'arrivent pas à suivre correctement leur traitement.

\section{-. Faible efficacité des ARV selon les PS}

Pour certains PS comme nous l'avons montré un peu plus haut, les ARV sont un traitement supposé d'apporter la guérison aux personnes séropositives au VIH. Pourtant, en dehors des ARV, d'autres médicaments sont parfois prescrits aux PS par les agents de santé en cas d'épisodes de pathologies opportunistes. Cette situation qui augmente les dépenses de santé des PS les indigne et les amène alors à s'interroger sur l'efficacité même des ARV. Une PS PDV le démontre à travers ces propos: "Malgré les médicaments de VIH et puis je paye médicaments au dehors là (hors du centre de santé, dans les officines privées), c'est que où est leur efficacité alors? C'est ce qui m'a découragée et puis je dis que je ne viens plus prendre les médicaments. Pour moi c'est une perte de temps ». (Une PS PDV du centre Espérance).

Pour eux, en effet, associer d'autres médicaments aux ARV signifierait que ces derniers sont peu efficaces à eux seuls, surtout que la guérison attendue n'est toujours pas manifeste. Cette faible efficacité des ARV s'explique par les préjugés dont les ARV sont l'objet, étant un des préjugés qu'ils provoquent des avortements.

\section{- Les ARV, cause des avortements spontanés selon les PS}

Une autre raison d'abandon du traitement avancée par les PS est liée à la procréation illustrée à travers les propos de cette PS PDV: "Moi-même j'ai besoin d'enfant. Depuis que j'ai commencé à faire le traitement, si j'ai eu un retard, ça s'enlève seul "(PS PDV du centre Espérance) En effet, pour certains PS, les avortements spontanés sont provoqués par la prise des ARV qui rend ainsi toute procréation impossible pour les femmes sous traitement. En d'autres termes, la procréation est incompatible avec le traitement ARV, 
ce qui signifie que toutes les femmes, en particulier les PS qui désireraient contracter une grossesse, devraient alors abandonner les ARV (Erwin, 1999).

Penser une telle chose serait, en réalité, ignorer l'existence et la portée même de la PTME (Protection de la Transmission du VIH de la Mère à l'Enfant) qui est une prophylaxie appliquée chez les femmes enceintes vivant avec le VIH. Cela met en exergue les difficultés liées à l'observance des ARV que nous allons relever au point suivant.

\section{II-1.2 : Les difficultés liées à l'observance du traitement ARV}

- Des comprimés de trop grande taille

En plus de tout ce qui précède, les PS avancent aussi comme raison d'arrêt du traitement ARV la taille trop grande des comprimés, ce qui se présente à eux comme un véritable problème. En effet, non seulement la prise des ARV est épuisante physiquement et moralement, mais aussi elle est parfois redoutée par les PS à cause de la difficulté à avaler les comprimés du fait de leur grande taille. Cette difficulté est exprimée ici par une PS retournée aux soins du centre Espérance de San Pedro qui rapporte les propos d'autres PS ayant arrêté leur traitement : «D'autres disent qu'ils ne peuvent pas avaler les médicaments ; les médicaments sont gros, gros tout ça ». (Une PS retournée aux soins du centre Espérance de San Pedro)

Une PS PDV du même centre atteste : "Ce n'est pas facile à avaler. Mais on fait avec » (Une PS PDV du centre Espérance de San Pedro).

\section{- La fatigue du traitement ARV}

L'abandon du traitement chez les PS sous traitement s'explique aussi par le fait que le VIH/SIDA est une maladie chronique et la quasi-totalité des PS sous ARV supportent difficilement cette chronicité de l'infection. Il y a chez ces derniers une lassitude qu'ils finissent par ressentir après plusieurs mois de traitement (Gouvernet, 2006). Pour eux, en effet, prendre des comprimés chaque jour et cela tout le temps pour le restant de la vie devient pénible et harassant. Cela se dégage des entretiens que nous avons eus avec ces PS PVVIH. Pour corroborer nos propos, voici ce que dit cette PS PDV du centre Espérance : «...Souvent aussi, tout le temps avaler, c'est travail. Ce n'est pas facile ».

L'expression " fatigue » est récurrente dans les discours des personnes interrogées. Ici, le traitement est vu comme un poids difficile à supporter et dont il faut se défaire pour avoir une certaine liberté ou délivrance. Elles ne prêtent plus attention alors à l'infection et finissent par prendre la décision d'arrêter le traitement. Arrêter pour mettre fin aux souffrances, arrêter pour ne plus être en train de penser à son statut surtout lorsqu'on n'en a pas informé ses proches. 


\section{- Les effets secondaires des ARV}

L'enquête a montré par ailleurs que les effets secondaires des ARV font que certains patients arrêtent le traitement dès l'entame. En fait, le traitement provoque chez certains PS le sommeil qui les empêche de vaquer convenablement à leurs activités. C'est ce que rapporte ce PDV: «Non seulement tu as sommeil pour quelqu'un qui se débrouille. Tu finis de prendre les médicaments tu as sommeil. Tu es obligé de dormir. Il faut souvent prendre les choses pour ne pas dormir. Il faut que je prenne du café, du thé, c'est pourquoi c'est difficile ». (PSH PDV de 44 ans célibataire de la Clinique de Confiance).

Les effets secondaires du traitement ont conduit cette PS PDV d'origine ghanéenne à arrêter son traitement : "Le médicament je le prenais ça me faisait un peu bizarre c'est-à-dire je n'arrivais pas à garder la salive normale. Il faut que je rejette, je rejette... Donc, quand je suis partie au Ghana c'est fini. Je me suis dit non enfin, je vais me reposer un peu ». (PSF PDV d'origine ghanéenne de la Clinique de Confiance).

\section{II-2. Les itinéraires thérapeutiques et la qualité de vie des PS}

La perception de la faible efficacité que les PS ont des ARV pourrait les amener parfois à abandonner ce traitement et à rechercher ailleurs un recours thérapeutique supposé plus efficace (Crane et $a l$., 2006). Une PS PDV du centre Espérance rapporte ici les propos d'une autre PS qui a recours à la médecine traditionnelle pour avoir suivi sans satisfaction le traitement ARV : «...si je prends tel autre médicament, je vais avoir la santé. Donc je préfère abandonner le traitement $A R V »$.

\section{II-2-1. L'influence des itinéraires de soins sur l'état de santé physique et psychologique}

Face au VIH et aux maladies opportunistes, les médecines moderne, chinoise, naturelle et traditionnelle sont simultanément ou l'une à la suite de l'autre pratiquées par les PS à l'effet d'obtenir la guérison. En effet, dès l'annonce de la sérologie positive, la PVVIH, sous le choc, opte dans un premier temps pour la médecine moderne eu égard à l'assurance et au réconfort psychologique que le personnel médical lui donne quant à sa prise en charge. Toutefois, eu égard à la chronicité de cette pathologie, à la difficulté éprouvée dans la prise des médicaments, à l'apparition de certaines maladies opportunistes, au risque de se faire identifier comme PVVIH et d'être stigmatisées, certaines personnes finissent par abandonner la médecine moderne au profit des autres types de médecine et de l'automédication. Ainsi, le rapport à la maladie, l'inefficacité perçue et les exigences spécifiques de la prise en charge de la maladie pour chaque type de traitement peuvent justifier les choix thérapeutiques des PVVIH. 


\section{- Le premier choix dans l'itinéraire de soins}

Le premier choix reste déterminant dans l'analyse des itinéraires thérapeutiques. Il situe sur la hiérarchisation des compétences des acteurs médicaux. Ainsi, le choix des médecines se fait en fonction de la construction de leurs compétences. Certains, théoriquement, ont fait le choix de l'automédication ou des médecines naturelles (pratiquée par les naturothérapeutes), chinoises et traditionnelles. D'autres, par contre, ont fait de la médecine officielle ou moderne, leur premier choix. Qu'est ce qui peut donc expliquer le retour aux soins des PS PVVIH+?

\section{- Motivations de retour aux soins}

Les motivations de retour aux soins sont observées chez les PS qui sont revenus dans la file active après un moment d'arrêt du traitement.

\section{II-2.2- . Dégradation de l'état de santé}

Les PS sous ARV connaissent souvent des moments d'arrêt de traitement qui se situent ici entre trois mois et deux ans pour des raisons évoquées ci-dessus. Pendant ce long temps passé loin des médicaments, l'on constate chez les PS un changement s'opérer au niveau de leur état de santé. C'est la rechute ou la réapparition des infections opportunistes qui sont, entre autres, le paludisme, le manque d'appétit et les maux de tête. Une PS du centre Espérance retournée aux soins qui a fait l'expérience décrit sa situation : «J'étais toujours malade. Tantôt c'est moi qui ai maux de ventre, les maux de tête. En tout cas, un peu de tout... Genre tout mon corps me fait mal on dirait quelqu'un qui a rhumatisme. Bon, souvent j'ai vertige, je n'ai pas l'appétit, tout ça-là, les maux de tête violents. Ma tête me fait mal, tout de suite, je prends médicament, ça se calme. Dès que la dose du médicament quitte, ça reprend comme zéro. Même j'ai eu genre fontanelle tout ça-là ».

Ce malheureux changement d'état pousse ainsi les PS à une « prise de conscience » qui les amène à prendre la décision du retour aux soins, nous indique cette PS retournée aux soins de la Clinique de Confiance : "Je vis actuellement chez ma maman. Pour régler mon souci, je tombe malade seulement. Ça peut aller deux jours, trois jours. Quand je décide d'aller me chercher (faire le travail de PS) pour résoudre mon problème, je tombe malade. C'est là je suis venue à la clinique ».

Cela veut dire que ces PS sont bien conscients des conséquences de l'arrêt du traitement. Pour eux en effet, l'arrêt des ARV peut donner la mort, ce qui pourrait compromettre l'éducation et l'avenir des enfants. Alors, à l'analyse, le retour aux soins est parfois effectué par rapport aux enfants pour qui la mère est peut-être le seul soutien à tous égards. 


\section{II-2.3- L'espoir d'un « bon médicament » contre le VIH}

Le retour aux soins des PS est aussi déterminé par l'espoir d'obtenir un jour le remède qui pourrait leur apporter la guérison. Selon eux en effet, un remède pourrait être trouvé un jour compte tenu des avancées actuelles de la science en la matière. Pour bénéficier de cet éventuel remède, il faut être en vie et pour être en vie, il faut suivre son traitement ARV qui est pour l'instant le seul traitement disponible. C'est cet espoir qu'évoque ici cette PS retournée aux soins du centre Espérance : "Peut-être si tu prends ton médicament et qu'on trouve un bon médicament un jour, ça pourrait te permettre d'être guérie totalement. Mais si tu n'as pas pu faire, peut-être que tu meures aujourd'hui et c'est le lendemain qu'on trouve le médicament qui pourrait te guérir totalement. Malheureusement, voilà toi, tu es déjà partie ».

\section{III- L'environnement social et la qualité de vie des professionnels du sexe}

\section{III-3-1. Les relations avec le personnel du centre}

Le personnel du centre de suivi joue parfois un rôle essentiel dans le processus de retour aux soins des professionnels du sexe. En effet, dans leurs échanges avec les PS PDV lors des rencontres, ils parviennent toujours à leur faire prendre conscience du fait qu'un arrêt du traitement peut les mener à la mort, d'où la nécessité pour eux de retourner au centre pour continuer leur traitement. Une PS retournée aux soins témoigne : « ... À chaque fois qu'elle (personnel de santé) me voit, elle me réprimande. Elle dit que si je fais comme ça-là, je vais perdre ma vie. J'ai réfléchi, je trouve que justement ce qu'elle a dit c'est la réalité» (Une PS retournée aux soins du centre Espérance).

Les motivations du retour aux soins suscitent également les motivations des auto-transferts au niveau des PS PVVIH+.

\section{III-3-2- Les relations avec le voisinage}

\section{- La crainte de stigmatisation ou de rejet}

Quel que soit l'espace social, force est de constater que les PS vivent dans un environnement très stigmatisant. Les personnes enquêtées vivent généralement dans "des cours communes », et généralement, dans ce type d'habitation, la moindre indiscrétion peut entraîner le dévoilement du statut sérologique. Pour cette raison cette PS PDV de la Clinique de Confiance préfère garder le secret de sa séropositivité : «Ça c'est moi-même mon secret. En disant ça à quelqu'un, un jour quand ça ne va pas entre vous, on va te le jeter à la figure... ».

La crainte d'être stigmatisés fait que les PS sous ARV refusent pour la plupart de dévoiler ou de partager leur statut avec leur entourage. En effet, en matière de VIH, l'infection peut non seulement affecter l'état de santé psychologique mais aussi entraîner des changements fondamentaux dans le 
mode de vie du professionnel du sexe. La crainte de la stigmatisation évoquée ci-dessus pose la question du silence autour duquel risque de s'organiser des non-dits, accentuant pour certains PS leur isolement. Cette PS PDV de la Clinique de Confiance, par exemple, a refusé de divulguer son statut. Elle est musulmane. Le père est nigérien et la mère ivoirienne. Au sein de sa communauté religieuse, les valeurs culturelles et sociales sont très intransigeantes tant à l'égard de la prostitution que du VIH qui est une infection qui se contracte généralement par voie sexuelle. C'est pourquoi à la question de savoir si elle a divulgué son statut, elle a une réponse qui est illustrative de la stigmatisation dont elle sera l'objet si elle dévoilait son statut : "J'arrive à gérer ça seule. ».(Cette PS PDV de la Clinique de Confiance). Il $\mathrm{y}$ a là une attitude d'isolement, de solitude. Cette PS préfère gérer l'infection toute seule.

\section{- La crainte du dévoilement du statut sérologique}

À travers les entretiens réalisés, nous avons constaté que la crainte que le statut sérologique soit dévoilé dans l'entourage est trèsvisible dans les comportements des PS. Les conduites ont donc toujours reflété la méfiance et la prudence (Achili et al., 2000). En effet, certains PS se cachent pour prendre leurs médicaments lorsqu'ils sont en présence des proches non informés de leur statut. Cette situation très gênante amène à la longue les PS à abandonner le traitement ARV pour éviter d'éventuels soupçons ou des questionnements. Cela est plus accentué chez ceux qui vivent en famille ou en couple. Une PS retournée aux soins du centre Espérance rapporte ici le cas d'une autre PS qui est dans une relation de couple : «Bon, elle dit qu'elle n'a pas dit à son monsieur. Elle ne lui a pas dit donc pour prendre c'est difficile. Elle dit que souvent, elle reste couchée avec son gars jusqu'à 8 heures, 9 heures pourtant elle doit prendre son médicament à 8 heures mais elle ne sait pas comment elle va faire pour prendre les médicaments... Donc, c'est quand il sort à 10 heures, 11 heures, 12 heures qu'elle prend. Donc, ça ne l'arrangeait pas... Elle a arrêté ».

Cette dame démontre clairement qu'elle craint d'être humiliée, stigmatisée. Cette crainte fait qu'elle ne divulgue pas son statut même à son époux. En clair, certains patients craignent de dévoiler leur statut de peur d'être stigmatisés. Cette crainte de dévoiler le statut sérologique compromet le meilleur suivi du traitement. Contrairement à cette PS qui craint d'être stigmatiser, certains PS sous ARV refusent même de reconnaître leur statut sérologique. 


\section{III-3-3. L'auto-perception}

\section{- Le déni du statut}

Le déni du statut renvoie au refus de reconnaître son statut sérologique bien qu'étant sous traitement ARV. En effet, certains PS vivant avec le VIH ne croient pas souvent au résultat du test du VIH lorsque qu'ils sont déclarés séropositifs. Cette conduite est parfois motivée généralement par l'absence des pathologies opportunistes qui sont les signes manifestes de l'infection. Cela veut dire que ne pas faire de maladie pourrait signifier pour le patient qu'il n'est pas séropositif. Cela lui donnerait même des raisons pour mettre en doute les résultats du laboratoire. Ce déni du statut induit indubitablement chez les PS l'abandon du traitement ARV. C'est le cas de cette PS retournée dans le groupe actif du centre Espérance qui venait à ses RDV au début du traitement parce qu'elle est inscrite, selon elle, dans le registre de prise en charge : "parce que je ne croyais pas. Donc, je n'ai pas suivi. Quand on me dit viens tu vas prendre, je dis d'accord. Je prends mais, j'envoie à la maison, je ne prends pas. Mais c'est arrivé un moment, je vois tantôt je suis couchée jusqu'à un mois, deux mois. Chaque un mois, deux mois, en tout cas, il faut que je sois à l'hôpital. Ils vont mettre sérum, c'est moi ils vont mettre sur le lit chaque fois. J'ai pensé, j'ai dit bon, est-ce que ce n'est pas ce qu'ils ont dit que j'ai ? Les médicaments que j'envoie et que je ne prends pas, est-ce que ce n'est pas ce qui fait ça-là par hasard? Bon attends, je vais essayer de reprendre de la manière qu'ils me l'ont expliqué».

L'acceptation du statut sérologique serait alors le starter nécessaire à tout bon suivi du traitement ARV au regard des propos de cette PS. En effet, il est évident que celui qui n'accepte pas son statut ne sera naturellement pas disposé à suivre convenablement le traitement d'une maladie qu'il se dit ne pas avoir.

\section{- L'auto-stigmatisation}

À la question de savoir pourquoi elle a arrêté le traitement, la PS PDV de la Clinique de Confiance a fondu en larmes. Être infecté du VIH est perçu comme une fatalité. Il y a comme un sentiment d'impuissance. Ce qui conduit la PS à dire que "le mal est déjà fait ".

Il y a une envie d'en finir avec la vie parce que pour elle ce mal ne peut être guéri. Il y a chez la PS donc ce que nous appellerons une "autostigmatisation suicidaire ». En effet, certains PS ont des envies de suicide bien que cette envie soit stoppée quelque fois à cause de l'amour maternel pour les enfants. C'est ce que semble dire d'ailleurs cette même participante : "Je voyais que le mal est déjà fait et un moment je voulais finir avec la vie. C'est vrai hein! (avec des pleurs dans la voix). Je ne voulais plus vivre! C'est à cause de mes enfants. J'ai eu pitié (en pleurs)» (PS PDV de la Clinique de Confiance). 
Cette auto-stigmatisation suicidaire s'est traduite malheureusement par la mort de cette PS PDV qui n'a pas accepté de prendre ses médicaments. Nous vous rapportons les propos de cette PS : "C'est arrivé à plusieurs personnes et j'ai vu. Des personnes comme moi que j'ai emmenées ici. Mais quand elles viennent prendre les médicaments, elles vont les cacher dans les maisons. Je suis fatiguée de leur parler. Je connais même une. Elle n'a pas voulu (prendre les médicaments) mais quand elle a rechuté, rien n'a été possible, elle est restée dedans, en laissant son enfant, nous avons fait ses obsèques» (PS PDV de la Clinique de Confiance).

Il y a donc cette auto-stigmatisation suicidaire qui conduit les PS à ne pas prendre leurs ARV, une volonté d'en finir avec leur vie. Ils perçoivent cette maladie comme une sorte de condamnation à mort, une sanction divine surtout, par rapport à l'activité qu'ils exercent.

\section{- La mobilité}

La mobilité est le déplacement généralement volontaire d'individus ou de populations d'un pays vers un autre ou d'une région vers une autre, pour des raisons économiques, politiques ou culturelles. Cette mobilité est caractéristique aux PS. En effet, elles sont présentes lors de grandes rencontres de toutes sortes (colloques, galas, grandes cérémonies) dans les grandes villes du pays (Abidjan, Yamoussoukro, Bouaké, San Pedro, Grand Bassam etc.). Cette mobilité est une exigence du métier en tout cas pour celui ou celles qui veulent se faire de l'argent. Mais, elle est un frein pour les PS séropositifs. Ces derniers n'arrivent pas à suivre leur traitement du fait de leur fréquente mobilité. C'est le cas de cette PS PDV du centre Espérance qui s'était rendue, dit-elle, auprès de sa mère malade : "Le voyage je suis partie et puis, c'était ma mère qui était malade. Donc, c'est à cause de la maladie de ma maman que je suis partie jusqu'à j'ai fait deux (2) ans là-bas avant que je revienne ». Quant à la seconde PS, elle a séjourné pendant cinq (5) mois à Niamey. Tout comme la première, la dégradation de son état de santé l'a amenée à revenir en Côte d'Ivoire pour se soigner : "Oui à Niamey ville c'est là-bas que j'étais. J'ai fait une petite formation là-bas. J'étais à Niamey mais j'ai fait juste on dirait cinq (5) mois. Je ne pensais pas durer» (Une PS retournée aux soins de la Clinique de Confiance).

En fait, le statut de séropositif participe à cette situation de mobilité. Les PS sous ARV éprouvent le besoin de voyager dans l'optique de se faire plus discret car plus ils voyagent plus l'on en sait moins sur leur statut. Il y a une volonté voire même une détermination de ne pas dévoiler son statut. En définitive, la mobilité est un frein à la prise des ARV et au suivi du traitement pour les PS séropositifs. Ils abandonnent leur traitement et généralement le retour à la clinique se fait lorsque leur santé s'est véritablement dégradée. Cette mobilité est caractéristique des PS qui vivent dans un état de précarité. 


\section{- La précarité ou le manque de moyens financiers}

De manière générale, la précarité apparaît comme une catégorie des populations dépourvues de revenu, d'accès à l'emploi, à la santé, au logement et ayant des difficultés de suivre une éducation adéquate. Elle se réfère aussi au " risque» de dégradation de la situation sociale des individus et à l'« incertitude » de leur parcours de vie (Bresson, 2007). Cette incertitude du parcours de vie est illustrative au niveau des populations hautement vulnérables que sont les professionnels du sexe, surtout dans les pays en voie de développement. Il y a donc à l'évidence, une sorte de précarité sociétale enracinée. Une précarité sociétale enracinée pour dire que la pauvreté est devenue plus que le quotidien des populations, nous dirions le compagnon des populations. Pour Oxford Policy Management (OPM, 2012), «les chocs économiques, sociaux et politiques qui ont touché la Côte d'Ivoire ces trente dernières années ont eu un impact majeur sur le bien-être de la population ivoirienne. Le taux de ménages pauvres a quadruplé entre 1985 et 2008, et les ménages déjà pauvres se sont encore appauvris. Les déficits en matière d'accès aux services essentiels tels que la santé et l'éducation se sont accrus, le phénomène de malnutrition s'est aggravé, et la participation à la vie économique des individus s'est affaiblie. Les inégalités se sont amplifiées, risquant de porter atteinte à une cohésion sociale déjà fragile ». Ces indices d'Oxford Policy indiquent le niveau de précarité des populations. Difficile donc d'évoquer les questions d'ordre sanitaire dans un tel contexte.

En effet, bien que bénéficiant de traitement gratuit dans les deux centres de santé (Clinique de Confiance et Centre Espérance), le niveau de précarité de certains PS fait qu'ils abandonnent le traitement. C'est le cas par exemple de cette PS PDV du Centre Espérance..: « C'est affaire de (frais de) transport. Si je sais que je n'ai pas transport je ne peux pas venir ». Ceci veut dire que certains PS ont la volonté de continuer leur traitement mais parfois, les moyens financiers leur font défaut et se voient ainsi contraints d'abandonner.

\section{- Les difficultés d'accès au centre de santé}

Nous avons constaté que la ville n'est pas le seul lieu du travail des PS. Il $\mathrm{y}$ a aussi les localités rurales où il $\mathrm{y}$ a une forte concentration de populations du fait des activités agricoles qui s'y développent. Malheureusement, force est de constater que les voies d'accès à ces localités sont dans un mauvais état puisque n'ont pas connu aucun reprofilage depuis un certain nombre d'années. De ce fait, les véhicules y arrivent rarement rendant ainsi le déplacement des populations difficile. L'on pourrait même passer plusieurs semaines sans y rencontrer le moindre véhicule. Cette réalité, qui est un véritable problème pour les $\mathrm{PS}$ sous $\mathrm{ARV}$ vivant à ces endroits souvent bien reculés, est traduite dans les propos de cette PS PDV. À la question «Quelles 
sont les raisons pour les PS d'arrêter le traitement ARV ? », elle répond ainsi : "Elle peut être loin. Par exemple, il y a d'autres qui sont où il n'y a pas de goudron; pour avoir un véhicule c'est dur...». (PS PDV du Centre Espérance) La rareté des moyens de transport en commun qu'évoque ici cette participante est source d'ennuis pour les PS, ce qui pourrait les démotiver à la longue et abandonner le traitement ARV.

\section{- Les croyances religieuses}

Les entretiens avec les PS nous montrent aussi que la religion peut avoir un rôle négatif sur le traitement ARV. En effet, dans leur quête de guérison, certains PS préfèrent se confier aux pasteurs et prophètes dans des églises ou camps de retraite pour des prières de guérison accompagnées de jeûne. Et cela au détriment des ARV justement. Pour eux en effet, Dieu peut guérir le VIH. Par la prière, ils peuvent obtenir la guérison que ne pourraient donner les ARV. C'est ce que montre ici cette PS retournée aux soins du Centre Espérance à travers cet exemple : "On a même eu un exemple ici. Il y a une dame (PS) qui est venue d'un campement. Elle dit qu'elle est allée quelque part, le prophète lui a dit non, il faut prier, jeûner pendant des jours pour que ça puisse aller. Elle ne prenait même plus ses médicaments, elle était là. Elle dégradait au fur et à mesure et puis elle est venue ici ». En acceptant d'observer le jeûne, les PS font fi du coup de certains conseils post-test qui leur ont été donnés par les agents de santé, notamment prendre régulièrement son traitement et bien se nourrir. Toute chose qui pourrait participer à la longue à la dégradation de leur état de santé.

\section{- Les incarcérations}

Pour ce qui est des emprisonnements, il faut relever que l'étude a montré que l'activité prostitutionnelle rime avec l'insécurité. Les professionnels du sexe, dans l'exercice de leur métier, sont victimes de toutes sortes de situation. Non seulement ils sont obligés de faire face aux colibris des populations riveraines mais aussi ils sont souvent victimes de racket de la part des forces de l'ordre. Parfois, ils sont raflés et conduits en prison. C'est le cas de ce PS PDV de la Clinique de Confiance : "Comme dans ces temps-ci même j'ai fait deux (2) mois sans prendre (les ARV) parce que j'ai été raflé avec des amis sur le trottoir. Ils nous ont emmenés jusqu'à la MACA (la prison)».

En somme, l'incarcération des PS est un déterminant social qui fait que les professionnels du sexe arrêtent souvent leur traitement.

\section{DISCUSSION}

La question du VIH-SIDA et des ARV chez les professionnels du sexe met en avant plusieurs facteurs (statut social, la culture, la religion, les rapports 
avec le corps médical et les agents sociaux, etc...). Chaque facteur joue du mieux qu'il peut son va-tout pour sauver ou sauvegarder la vie du PS. Mais lors de nos enquêtes, une agente de santé lors d'un échange informel a fini par avoué ceci « le VIH-SIDA est une sale maladie et prions Dieu de ne jamais être infecté. L'infecté est déjà abattu mentalement quelques soit ce que nous lui disons. En plus tous les infectés n'ont pas le même organisme. Certains organismes ne supportent pas les ARV à la longue et finissent par succomber à notre corps défendant $\gg$.

L'approche stratégique et « jeu » des acteurs au regard des propos de cette responsable communautaire de santé et régulièrement en contact avec les PS nous situe sur l'état de santé des PS et singulièrement des PS. En effet, selon la constitution de l'OMS, la santé est définie comme à "un état complet de bien-être physique, mental et social et pas simplement une absence de maladie et pas ou d'infirmité ". Au regard de cette constitution de la santé, les PS sous ARV ne peuvent pas l'atteindre. Nous avons en face de nous des individus que nous appellons d'un point de vu socio-anthropologique « des PS sous ARV handicapés ». En effet, le VIH-SIDA se guérit mais ne se soigne pas. Vous êtes malade à vie. Une fois infectée vous « devez faire avec jusqu’à votre mort. Vous ne pouvez pas aussi « cacher la maladie » confesse un PS sous ARV sous anonymat. Il ajoute que «c'est pire qu'une condamnation à vie $\gg$.

Pour dire que les offres de soin et la qualité de vie des professionnels (les) du sexe sous ARV œuvrent à maintenir certes les PS sous en ARV en meilleure santé mais pour la plupart ces soins ne font que repousser « une sentence connue d'avance » pour pasticher les propos d'un pair de PS sous ARV.

La question du VIH-SIDA et des PS sous ARV au bout du compte montre leur vulnérabilité et leur handicap. Un handicap d'abord biologique parce que la maladie est présente dans leur sang, un handicap psychologique car il faut vivre avec la maladie et un handicap social et culturel parce que nos sociétés africaines fondamentalement communautaires gardent toujours l'image d'une société axée sur les notions de pudeur, de sacralité du sexe et d'un attachement sacro-saint au valeur religieuse, pour dire qu'elle rejette systématiquement tout individu infecté du VIH-SIDA et de surcroit PS. Difficile donc de surmonter ce triple handicap à la fois biologique, psychologique et socio-culturel.

\section{Conclusion}

Le VIH/SIDA continu d'endeuiller de nombreuses familles en Côte d'Ivoire. Plus spécifiquement à Marcory, dans le milieu des professionnelles du sexe, cette maladie est bien connue et fait des victimes. Tout compte fait, les personnes atteintes dans ce milieu ont conscience de l'existence de soins 
appropriés. En fait, elles connaissent et fréquentent pour la plupart les centres en charge de ce mal. Cependant, leurs perceptions de ces soins influencent considérablement leur prise en charge, et même si certains malades reconnaissent l'efficacité des traitements, ils brandissent la stigmatisation perpétuelle dans leur cadre de vie et de travail lorsqu'ils sont identifiés en tant que tels.

Les attitudes et comportements de ces derniers sont généralement un frein à leur guérison et plus encore à la réduction de la maladie. Ce milieu qui est pratiqué de plus en plus par toutes les catégories de jeunes a réellement besoin d'être sensibilisé quant à l'enjeu de la prise en charge. D'une part, les malades avec leurs différents profils sont source de contagion pour leur propre famille (mères, parents) et d'autre part pour la clientèle qui ignore leur sérologie. Ne l'oublions pas, les rapports non protégés sont affectionnés par certains clients qui n'hésitent pas à payer au-dessus des prix négociés.

Malgré tout, une approche plus spécifique devrait permettre de voir la maladie comme toute autre maladie pour encourager les malades à se soigner. Si leurs représentations sociales de la maladie influencent leur choix de se traiter en tenant compte aussi des effets indésirables de certains médicaments, il serait judicieux d'introduire les programmes au sein d'autres maladies comme le paludisme, la tuberculose pour limiter la stigmatisation.

\section{References:}

1. Achili, L. \& Hejoaka, F. (2000). Les associations dans la réponse à l'épidémie VIH/sida au Burkina Faso.

2. Balde, F. (2013). L'abandon du traitement ARV chez les femmes vivant avec le VIH /sida en Guinée. Mémoire, Université du Québec

3. Barbara, P. (2000). "Association de lutte contre le sida à Ouaga: contexte d'émergence, profils et pratiques. " mémoire DESS pratiques sociales du développement, actions locales et sectorielles. Paris I

4. Carillon, S. (2010). Ruptures de suivi médical en milieu africain: l'exemple de Kayes. Mémoire Master, Université Paris 5

5. Crane, J.T., Kawuma, A., Oyugi, J.H., Byakika, J.T., Moss, A., \& Bourgois, P. (2006). "The price of adherence: qualitative findings from HIV positive individuals purchasing fixed-dose combination generic HIV antiretroviral therapy in Kampala Uganda». AIDS and Behavior, vol. 10, n 4 , pp. 437-442.

6. Cuchet, F. (2011). étude des répercussions de l'indigence sur le suivi des PVVIH sous traitement ARV au centre de traitement agréé de l'hôpital Laquintinie de Douala. Thèse médecine. Université de Grenoble 
7. Erwin, J. \& Barry, P. (1999). «Treatment issues for HIV+Africans in London». Social Science \& Medicine, vol. 49, n¹1, p. 1519-1528.

8. Gouvernet, B. \& Viaux, JL. (2006). "Emprise et relation aux traitements antirétroviraux: Emprise and relation to antiretroviral treatment ». AnnalesMédico Psychologiques, vol. 164, nº, p. 470475.

9. Gruenais, M. (1999). «Un marché du sida » in Vivre et penser le sida en Afrique. Codesria-Karthala-IRD. Dakar-Paris.

10. Olivier, F., N'Kam, M., Midoungue, D. \& Rey, J.L. (2005). «Etude sur l'observance des traitements antirétroviraux au Centre Hospitalier Universitaire de Yaoundé (Cameroun)». Santé publique, vol. 4, $\mathrm{N}^{\circ} 17$, p. 559-568. 